\title{
Do Therapists Cry in Therapy? The Role of Experience and Other Factors in Therapists' Tears
}

\author{
Amy C. Blume-Marcovici, Ronald A. Stolberg, and Mojgan Khademi \\ Alliant International University
}

\begin{abstract}
The subject of therapist's crying in therapy (TCIT) has been virtually ignored in the literature, with only 1 qualitative dissertation and 3 case studies devoted to the topic. This mixed-method survey study explored therapists' experiences with and attitude toward TCIT. Six hundred eighty-four U.S. psychologists and trainees filled out the survey online, revealing that $72 \%$ of therapists report having cried in therapy in their role as therapist. Data analysis indicated that the act of crying in therapy has less to do with personality or demographic factors (i.e., Big Five traits, empathy, sex) and more to do with the unique aspects of the therapy itself and the therapist's identity in the therapeutic context (theoretical orientation, clinical experience, affective tone of the session). Clinicians with more experience, who are older, cried more in therapy than novice clinicians, despite lower crying frequency in daily life, suggesting that more experienced therapists feel more comfortable allowing themselves to experience and/or express such emotions in therapy sessions. Psychodynamic therapists reported slightly higher rates of TCIT than cognitive-behavioral therapists despite no differences in crying in daily life. Despite significant differences in crying rates in daily life, male and female clinicians report similar rates of TCIT. Data regarding the relationship between TCIT and Big Five personality traits, empathy, and perceived consequences of TCIT are reported.
\end{abstract}

Keywords: therapist crying, tears, emotional expressions, crying in therapy

A therapist's responses to his or her client during a psychotherapy session are important for the course of treatment and therapeutic rapport (Kahn \& Fromm, 2001; Summers \& Barber, 2010). Logically, we can extend this idea to include the notion that a therapist's emotional responses to his or her client during a psychotherapy session are, similarly, important in treatment. Yet, very little research has explored the role of a most fundamental expression of human emotion by the therapist in the therapeutic setting: that is, the role of the therapists' crying in therapy (hereafter, TCIT).

Passing references to therapist crying can be found throughout psychological literature. For instance, Nancy McWilliams (1994), in a footnote, justifies the use of the psychoanalytic couch to hide one's tears:

(the couch) allows the therapist the freedom to respond internally to the patient's material without self-consciousness: to fantasize, to respond affectively, even to weep without worrying that the patient will be distracted from the internal processes by the therapist's emotional reactivity (p. 242).

In a case study, psychologist Phyllis Alden (2001), describing a transformative therapy session, recalled the feeling of her own

This article was published Online First February 11, 2013.

Amy C. Blume-Marcovici, Ronald A. Stolberg, and Mojgan Khademi, Department of Clinical Psychology, Alliant International University.

Thank you to Dr. Matthew Porter for his valuable feedback on this project.

Correspondence concerning this article should be addressed to Amy C. Blume-Marcovici, Alliant International University, Department of Clinical Psychology, 10455 Pomerado Road, San Diego, CA 92131. E-mail: acb46@columbia.edu tears during that session, flowing down her cheeks. Similarly, in a case excerpt in their text on psychodynamic psychotherapy, Summers and Barber (2010) write, "When (the patient) told me about the sudden death, I teared up, experiencing these losses with him" (p. 239). And Harry Guntrip (1975/1986), in his account of being in analysis with W. R. D. Fairbairn, also references his famous therapists' tears:

"As I was finally leaving Fairbairn after the last session, I suddenly realized that in all the long period we had never once shaken hands, and he was letting me leave without that friendly gesture. I put out my hand and at once he took it, and I suddenly saw a few tears trickle down his face. I saw the warm heart of this man with a fine mind and a shy nature" (p. 454).

However, none of these writers elaborate further on these seemingly poignant moments in their therapies.

Such vague references to crying create a mystery around the phenomenon of TCIT, a mystery further substantiated by the dearth of literature on the topic. A literature search with the words "therapist crying" in the PsycInfo database returns one hit: a qualitative dissertation conducted more than 15 years ago (Waldman, 1995). A search for the terms "psychologist crying" or "therapist tears" returns no results.

Three case studies explicitly write of TCIT (Counselman, 1997; Owens, 2005; Rhue, 2001), revealing themes of clinicians' conflict about their tears, with fears that TCIT may breach professionalism or harm the client. The authors, however, largely conclude that their TCIT was therapeutically appropriate or even beneficial in the treatment. In the one qualitative research project devoted to TCIT, Jane Waldman (1995) interviewed 10 psychodynamic psychotherapists who had cried during therapy in their role as psy- 
chologists. All but one of the subjects felt that their TCIT had been useful to the treatment, communicating genuineness and/or facilitating the client's ability to express affect. Additionally, in an article on therapeutic immediacy, Mayotte-Blum et al. (2012) highlight a case segment in which a therapist cries. These authors discuss the importance of TCIT in providing an opportunity for therapeutic immediacy, in that TCIT leads to an open discussion between therapist and client about the therapists' affect and its impact on the client. Specifically, the client describes feeling "reassured" and as if she is "being joined" (p. 35) by the therapist in her sadness because of his tears. Although these authors elaborate on the meaning of TCIT in this therapy moment, TCIT was not the explicit focus of the study.

One survey on ethical issues in psychological practice (Pope, Tabachnick, \& Keith-Spiegel, 1987) included a single item on therapist crying in the presence of a client. In this study, $56.5 \%$ of the 456 respondents reported that they had cried in the presence of a client. This data point provides the only existing estimate of the percentage of therapists who cry in therapy.

Aside from an explicit focus on TCIT, several studies (Labott, 2001; Nelson, 2008; Van Heukelem, 1979) have investigated clients' tears in therapy, and have even addressed therapists' attitude toward and responses to client crying. These studies have suggested that how the therapist responds to the crying patient is important for therapeutic outcome (Labott, 2001). They have also suggested that one possible response to client crying is a therapist's own tears (Nelson, 2008; Van Heukelem, 1979). However, no study has dedicatedly investigated how frequently such a response occurs or the context in which it may occur.

Compared with the dearth of empirical studies on professionals' tears in psychological literature, medical professionals have published multiple surveys, one case study, and several "op-ed" news pieces on crying behaviors of medical care providers (Barth, Egger, Hladschik-Kermer, \& Kropiunigg, 2004; Blankenship, 1984; Krauser, 1989; Kukulu, 2006; Lerner, 2008; Majhail \& Warlick, 2011; Sinclair, 2011; Sung et al., 2009; Wagner, Hexel, Bauer, \& Kropiunigg, 1997). It is striking that, as a profession that so explicitly values emotions and interpersonal interaction, psychologists have attended less to the topic of tears than medical professionals.

The purpose of the present study was to address this gap in the literature by empirically investigating TCIT within the psychology community. Specifically, this project investigated when and how often therapist tears occur in session, demographic and clinical correlates of therapist tears, and any perceived therapeutic value or cost. The aim of this effort was to deepen the field's understanding of therapists' emotions and emotional expressions in the clinical context, not only to further our knowledge about such ephemeral moments in psychotherapy, but also to help therapists think about and predict their own likelihood of TCIT and prepare for how to handle their tears if and when they occur.

\section{Method}

\section{Participants}

Six hundred eighty-four U.S. psychologists, postdoctoral psychology fellows, and psychology graduate students participated in this study by completing at least part of the survey. Of these, 541
(79\%) participants completed the survey in full. To represent all of the individuals who participated in the study, even in part, data are included when available for each question.

In terms of sex, $75 \%(n=515)$ of respondents were women and $25 \%(n=169)$ were men (total $N=684)$. Ethnically, $80 \%(n=$ $523)$ of respondents identified as non-Hispanic White, 5\% $(n=$ 36) as Asian or Asian American, 5\% $(n=31)$ as Hispanic or Latino, and 3\% $(n=18)$ as Black or African American. The rest of other ethnicities made up 7\% $(n=42)$ of respondents. The mean age of respondents was 36 years, and the median age was 32 years. Age ranged from 22 to 85 years (total $N=643$ ). At least 40 U.S. states were represented (total $N=551$ ).

In terms of professional status, 57\% $(n=390)$ of respondents were graduate students in doctoral programs in psychology, $40 \%$ $(n=273)$ were licensed psychologists, and 3\% $(n=21)$ were postdoctoral fellows or license-eligible psychologists (total $N=$ 684). Of the licensed psychologists, the mean number of years licensed was 9.6, the median was 6 years, and the range from less than 1 to 33 years.

In terms of theoretical orientation, 35\% $(n=232)$ of respondents identified as cognitive-behavioral therapists, $23 \%(n=157)$ as eclectic/integrative with a psychodynamic emphasis, $19 \%(n=$ 129) as eclectic/integrative without a psychodynamic emphasis, $11 \%(n=76)$ as psychodynamic, $10 \%(n=68)$ as "other," and $1 \%(n=9)$ as psychoanalytic (total $N=671)$.

\section{Measures}

All participants were asked to complete the Ten-Item Personality Inventory (TIPI; Gosling, Rentfrow \& Swann, 2003), a very brief measure of personality based on the Big Five model of personality. The Big Five model classifies human personality into five bipolar categories: extraversion (i.e., outgoing/energetic), agreeableness (i.e., cooperative/empathic), conscientiousness (responsible/orderly), neuroticism (tendency to experience negative emotions/easily upset), and openness to experience (attentive to inner feelings/curious) (John \& Srivastava, 1999). Some sacrifices, particularly in terms of reliability and validity, were made in using such a brief (10 item) personality measure. Interitem correlations for the TIPI, for instance, are somewhat low, with a mean $r$ of .55. However, convergent validity of the TIPI with longer validated measures of the Big Five traits, such as the Big Five Inventory, are considerably high, with a mean $r$ of .77 ( $p<$ .01). Discriminant validity correlations are much lower (mean $r=$ .20 ), as one would hope. Test-retest correlations for the TIPI are also substantial, with a mean $r$ of .72 (Gosling et al., 2003). Raw means and standard deviations of TIPI scores from the study sample are presented in Table 1.

Participants also completed the Toronto Empathy Questionnaire (TEQ; Spreng, McKinnon, Mar, \& Levine, 2009), a brief unidimensional measure of empathy with strong internal consistency (Cronbach's alpha $=.85)$ and demonstrated concurrent $(r=-.30$, $p<.001)$ and convergent $(r=.80, p<.001)$ validity (Spreng et al., 2009). The TEQ consists of 16 statements about situations in which one may experience empathy. Participants describe their own typical response to such situations by marking "never," "rarely," "often," or "always." The TEQ defines empathy as primarily an emotional process based on affective insight into the feeling state of another. Raw means and standard deviations of TEQ scores from the study sample are presented in Table 2. 
Table 1

TIPI Means and Standard Deviations for the Present Sample ${ }^{\mathrm{a}}$

\begin{tabular}{|c|c|c|c|c|c|c|}
\hline Sample & $\begin{array}{c}\text { Emotional } \\
\text { stability }\end{array}$ & Extraversion & Agreeableness & Conscientiousness & Openness & $N$ \\
\hline Total sample & $5.13(1.18)$ & $4.51(1.53)$ & $5.67(0.98)$ & $5.97(1.00)$ & $5.54(1.04)$ & 650 \\
\hline Women & $5.03(1.14)$ & $4.59(1.51)$ & $5.72(0.93)$ & $6.03(0.96)$ & $5.53(1.02)$ & 490 \\
\hline Men & $5.42(1.23)$ & $4.27(1.58)$ & $5.54(1.10)$ & $5.79(1.10)$ & $5.58(1.12)$ & 160 \\
\hline Trainees & $4.99(1.78)$ & $4.46(1.50)$ & $5.68(0.95)$ & $6.00(0.96)$ & $5.51(1.01)$ & 369 \\
\hline Licensed psychologists & $5.32(1.45)$ & $4.58(1.52)$ & $5.65(1.03)$ & $5.93(1.05)$ & $5.54(1.09)$ & 261 \\
\hline Dynamically oriented & $5.23(1.08)$ & $4.29(1.57)$ & $5.97(0.91)$ & $5.99(0.97)$ & $5.75(0.94)$ & 232 \\
\hline CBT & $5.06(1.20)$ & $4.51(1.55)$ & $5.55(1.04)$ & $6.01(1.00)$ & $5.28(1.06)$ & 225 \\
\hline Nonclinical sample (Gosling et al., 2003) & $4.83(1.42)$ & $4.44(1.45)$ & $5.23(1.11)$ & $5.40(1.32)$ & $5.38(1.07)$ & 1,813 \\
\hline
\end{tabular}

${ }^{\text {a }}$ Data are presented as raw means with standard deviations in parenthesis.

In addition, participants completed the Adult Crying Inventory-Short Form (ACI; Vingerhoets \& Cornelius, 2001), a validated measure comprising 17 statements describing situations and mood states that may elicit crying and two questions about past crying behavior, yielding scores for crying tendency (i.e., selfassessed predisposition toward crying), frequency (i.e., selfassessed number of actual crying events within a given period), and proneness (i.e., self-assessed likelihood or propensity of an individual to cry in a particular situation or context). The ACI Proneness scale is further broken down into two subscales: the ACI Positive Affect Proneness scale (proneness to cry owing to positive emotions, i.e., feeling happy) and the ACI Negative Affect Proneness scale (proneness to cry owing to negative emotions, i.e., feeling sad). Reliability and factor analysis investigating the underlying dimensionality of the ACI was conducted by Scheirs and Sijtsma (2001) using 3,547 data records. Using a Principal Components Analysis, the authors came to two conclusions. One found the ACI to have three factors, made up of distinct items on the ACI: crying from Distress, Sadness, or Joy, with alpha coefficients $>.8$ for each scale. The second analysis found two factors: crying from Unpleasant or Pleasant Emotions. The correlation between these two factors was .53. Raw means and standard deviations of ACI scores from the study sample are presented in Table 3.

Finally, participants completed the TCIT Survey 1, a 40-item survey created by the research team to gather information about whether participants had cried in therapy. This survey yielded information on demographics and therapists' beliefs about TCIT as well as data on several scales: the TCIT Tendency scale (based on the question, "In my role as a therapist, I have cried during

Table 2

TEQ Means and Standard Deviations for the Present Sample

\begin{tabular}{llr}
\hline \multicolumn{1}{c}{ Sample } & \multicolumn{1}{c}{ Mean $(S D)$} & $N$ \\
\hline Total sample & $44.94(4.22)$ & 626 \\
Women & $45.42(4.08)$ & 474 \\
Men & $43.46(4.33)$ & 152 \\
Trainees & $45.15(4.35)$ & 355 \\
Licensed psychologists & $44.54(4.00)$ & 252 \\
Dynamically oriented & $45.15(4.27)$ & 223 \\
CBT & $44.50(4.24)$ & 216 \\
Clinical sample (Cusi, MacQueen, & $61.4(7.2)$ & 20 \\
$\quad$ Spreng, \& McKinnon, 2011) & & \\
Nonclinical sample (Spreng et al., 2009) & $44.54(7.70)$ & 200 \\
\hline
\end{tabular}

therapy"; Likert scale of 1-5, where $1=$ I have never cried in therapy and $5=$ I cry very often in therapy), the TCIT Proneness scale (comprised of 16 survey items on which respondents rated their proneness to cry in specific clinical scenarios (e.g., "upon witnessing a client's suffering"; "terminating with a longstanding client"), and the TCIT Frequency Ratio (equated by dividing the self-reported number of TCIT episodes in the past 4 weeks by the self-reported total number of therapy sessions in the past 4 weeks). Additionally, frequency rates of only those therapists who reported crying in therapy were captured with the Affirmative TCIT Frequency Ratio, which was calculated in the same way as the TCIT Frequency Ratio but included only those who had previously cried in therapy. Those participants who reported having cried in therapy also completed TCIT Survey 2, a 49-item survey created by the research team to gather information about past experiences of TCIT, including questions regarding perceived therapeutic effects of TCIT (e.g., "Do you think your crying changed your relationship with your client?"). Survey questions were primarily in Likert scale format, although multiplechoice and checklist questions were also used. The TCIT Surveys 1 and 2 were piloted on a group of 20 psychology graduate students before the start of the study, and feedback was incorporated into final surveys.

\section{Research Questions, Design, and Procedures}

The present study, conducted as part of a doctoral dissertation project (Blume-Marcovici, 2012), aimed to answer eight specific research questions. The first was, simply, do therapists cry in therapy? To answer this question, the TCIT Tendency, TCIT Frequency, and Affirmative TCIT Frequency scales described earlier in the text were used.

Second, the research team sought to discover whether individuals with greater crying frequency, tendency, and proneness in daily life cry more in therapy (i.e., do criers cry?). To answer this, baseline rates of participants' crying, obtained through the ACI (Vingerhoets \& Cornelius, 2001), were compared with TCIT rates. The researchers hypothesized that there would be a significant and positive correlation between crying in daily life and crying in therapy.

A third research question asked whether there is a relationship between gender and crying. Differences in both ACI and TCIT scale scores of men and women were analyzed, and researchers hypothesized there would be a significant difference in both ACI 
Table 3

ACI Means and Standard Deviations for the Present Sample ${ }^{\mathrm{a}}$

\begin{tabular}{|c|c|c|c|c|}
\hline Sample & $\begin{array}{c}\text { ACI } \\
\text { tendency }\end{array}$ & $\begin{array}{c}\text { ACI } \\
\text { frequency }\end{array}$ & $\begin{array}{c}\mathrm{ACI} \\
\text { proneness }\end{array}$ & $N$ \\
\hline Total sample & $2.92(0.73)$ & $3.36(4.87)$ & $3.36(1.07)$ & 606 \\
\hline Women & $3.08(0.73)$ & $3.85(4.26)$ & $3.66(0.93)$ & 464 \\
\hline Men & $2.38(0.74)$ & $1.75(2.70)$ & $2.39(0.94)$ & 142 \\
\hline Trainees & $3.00(0.81)$ & $3.70(5.46)$ & $3.43(1.07)$ & 344 \\
\hline Licensed psychologists & $2.79(0.74)$ & $2.89(4.01)$ & $3.21(1.07)$ & 243 \\
\hline Dynamically oriented & $2.85(0.68)$ & $4.31(4.94)$ & $3.31(1.07)$ & 216 \\
\hline CBT & $2.77(0.81)$ & $2.60(3.37)$ & $3.19(1.05)$ & 209 \\
\hline Nonclinical U.S. sample (Becht, Poortinga, \& Vingerhoets, 2001) & - & $2.7(-)$ & $2.75(-)$ & $>30$ \\
\hline
\end{tabular}

${ }^{a}$ Data are presented as raw means with standard deviations in parenthesis.

and TCIT scores, with women having significantly higher scores than men.

A fourth research question asked whether certain personality traits are related to TCIT scale scores. To answer this, the TIPI (Gosling, Rentfrow \& Swann, 2003) was administered, and correlations between TCIT rates and Big Five personality traits were performed. The researchers hypothesized that the traits of both neuroticism and extraversion would significantly positively correlate with TCIT rates.

Next, the research team asked whether there is a relationship between empathy and rates of TCIT. To answer this, the TEQ (Spreng, McKinnon, Mar \& Levine, 2009) was administered, and correlations between empathy scores and TCIT scale scores were performed. The hypothesis was that there would be a significant positive correlation between TEQ and TCIT scores.

The next research questions evaluated differences in TCIT scale scores based on theoretical orientation and clinical experience to determine any relationships between crying, theoretical orientation, and level of clinical experience. The researchers hypothesized that psychodynamic clinicians would have higher TCIT rates than cognitive-behavioral therapists, but that there would be no difference in TCIT rates based on level of clinical experience. The final research question regarded the potential impact TCIT had on the clinical encounter, based on therapists' beliefs and experience.

The study used a single, cross-sectional, retrospective, selfreport Internet survey that incorporated questions developed by this researcher, as well as validated assessment measures, as described earlier in the text. A recruitment e-mail, with a hyperlink to the survey, was sent to university program directors, psychology training sites, and psychology associations throughout the United States. This e-mail explained that the study focused on therapist emotions in therapy (not crying specifically), and participants were asked to give their consent to participating by checking "yes" or "no" on a consent form.

Crying in this study was defined following the ACI (Vingerhoets \& Cornelius, 2001): "tears in one's eyes due to emotional reasons."

\section{Results}

\section{Do Therapists Cry in Therapy?}

Based on the TCIT Tendency scale, $72 \%(n=411)$ of therapists reported having cried in therapy, whereas $28 \%(n=157)$ reported never having cried in therapy $(N=568)$. Evaluating only those respondents who reported crying, $30 \%$ had cried in therapy during the past 4 weeks $(n=179)$. Although therapists who cried in therapy only cried in approximately $6.64 \%$ of their therapy sessions (Affirmative TCIT Frequency Ratio). Including those who reported never having cried, respondents reported crying in approximately $1.7 \%$ of therapy sessions over the past 4 weeks (TCIT Frequency Ratio).

\section{Do "Criers" Cry in Therapy?}

To compare crying frequency, tendency, and proneness in therapy (i.e., TCIT) with crying frequency, tendency, and proneness in daily life (i.e., to answer the research question, "Do therapists who cry more in daily life also cry more in therapy?"), scales from the ACI (Vingerhoets \& Cornelius, 2001) were analyzed in relation to TCIT scales. There was a significant and positive correlation between the TCIT Tendency scale and the ACI Frequency scale $(r=.192, p<.001, N=568)$, the ACI Tendency scale $(r=.298$, $p<.001 ; N=568)$, and the ACI Proneness scale $(r=.318, p<$ $.001 ; N=568)$, such that those who reported a greater frequency, tendency, and/or proneness to cry in daily life were more likely to report a greater tendency to cry in therapy. The strength of these correlations were small, medium, and medium to large, respectively. ${ }^{1}$

There was a significant and positive correlation between ratings on the TCIT Proneness scale and the ACI Tendency $(r=.270, p<$ $.001, N=403)$, ACI Proneness $(r=.363, p<.001, N=403)$, and ACI Frequency $(r=.235, p<.001, N=403)$ scales, such that the higher the tendency, proneness, and frequency of a respondent to cry in daily life, the greater their self-reported proneness to cry in therapy.

There was a significant and positive correlation between the TCIT Frequency Ratio and the ACI Frequency scale $(r=.179$, $p<.001, N=604)$, the ACI Tendency scale $(r=.167, p<.001$, $N=604)$, and the ACI Proneness scale $(r=.129, p=.001, N=$ 608 ), such that as crying frequency, tendency, and proneness in

\footnotetext{
${ }^{1}$ Hemphill (2003) found that the lower third of correlation coefficients in a large sample of treatment studies ranged from -.08 to .17 , the middle third from .17 to .28 , and the upper third from .29 to .60 . Thus, the author argues that Cohen's (1988) suggestion that correlation coefficients of approximately .10 are "small," those of approximately .30 are "medium," and those of approximately .50 are "large" may be too high for clinical samples.
} 
daily life increased, so too did crying frequency in therapy. However, these correlations were small.

\section{Does Gender Matter?}

Contrary to the researchers' expectations, there were no significant differences between men and women on the TCIT Tendency, TCIT Frequency, or TCIT Proneness scales.

Exploratory analyses found a small but significant gender difference regarding having resisted the impulse to cry in a therapy session, with women reporting a somewhat greater frequency of having resisted the impulse to cry in therapy than men $(81 \%$ of women $[n=452, M=1.19, S D=0.396]$ vs. $67 \%$ of men $[n=$ $139, M=1.33, S D=0.472])$. Thus, the gender difference is small but statistically significant $(F=11.44, d f=1, p=.001, d=$ 0.32). Among both men and women who had resisted the impulse to cry, the most common reasons for resisting were "I thought it would be harmful to the client" (87\%) and "I did not think it was professional" (45\%). Finally, there were some slight gender differences in how men and women answered a question about longevity of TCIT within the session, with men $(n=74, M=$ $1.73, S D=0.765)$ reporting longer periods of crying when they do cry than women $(n=288, M=1.45, S D=0.804)$. This difference was small, but statistically significant $(F=7.20, d f=1, p=.008$, $d=0.36$ ).

\section{Does Personality Matter?}

No clear trends regarding personality, as measured by the TIPI (Gosling et al., 2003), and TCIT were found. The personality traits of openness, agreeableness, and extraversion were significantly and positively related to TCIT Tendency $(r=.142, p=.001, N=$ 568; $r=.132, p=.002, N=568 ; r=.11, p=.009, N=568$, respectively), such that respondents with higher scores on the openness, agreeableness, and extraversion scales of the TIPI also reported a greater tendency to cry in therapy. However, these correlations were small and no correlations were found with TCIT Frequency or TCIT Proneness.

It is notable that although the personality trait of neuroticism significantly and positively correlated with crying in daily life, as measured by the ACI (neuroticism and ACI Tendency: $r=.248$, $p<.001, N=606$; neuroticism and ACI Proneness: $r=.297, p<$ .001, $N=610$; neuroticism and ACI Frequency: $r=.157, p<$ $.001, N=606$ ), there was only one very small correlation between neuroticism and TCIT: the greater the number of times a respondent reported crying in the past 4 weeks (TCIT Frequency Ratio), the higher their neuroticism score $(r=.099, p=.012, N=648)$. However, this correlation was very small, and no significant relationship was found between neuroticism and TCIT Tendency or TCIT Proneness.

\section{Does Empathy Make Us Cry?}

No clear trends regarding empathy and TCIT were found in the present study. Although rates of empathy, as measured by the TEQ (Spreng et al., 2009), significantly and positively correlated with TCIT Tendency ( $r=.125, p=.003, N=568$ ), no significant relationship was found between TCIT Frequency or TCIT Proneness. Thus, the relationship between empathy and TCIT remains equivocal. However, the relationship between empathy and crying in daily life was more pronounced, as TEQ scores significantly and positively correlated with the ACI Tendency scale $(r=.216, p<$ $.001, N=606)$ and the ACI Proneness scale $(r=.222, p<.001$, $N=610)$.

\section{Theoretical Orientation and TCIT}

An analysis of variance was conducted to evaluate differences in TCIT Tendency between theoretical orientations. The omnibus $F$ was significant $(F=10.543, d f=4, p<.001)$, and Scheffé post hoc planned comparisons found that cognitive behavioral therapy (CBT) clinicians were significantly lower in TCIT Tendency than all other theoretical orientations. To evaluate CBT versus dynamic clinicians more discretely, all dynamically oriented clinicians (psychodynamic, eclectic/integrative with a dynamic emphasis, and psychoanalytic) were combined in one category and compared with CBT therapists. The mean TCIT Tendency score for the aggregated-dynamic group $(n=242, M=2.14, S D=0.814)$ was significantly higher than the CBT group mean $(n=232, M=1.71$, $S D=0.665)$, with psychodynamic clinicians reporting a significantly higher crying tendency in therapy than CBT clinicians $(F=$ 32.984, $d f=1, p<.001, d=0.58)$.

In comparing frequency means of each theoretical orientation, psychoanalysts had the highest TCIT Frequency Ratio, crying in $3.3 \%$ of sessions, whereas CBT therapists had the lowest, crying in $1.3 \%$ of sessions (Table 4 ).

As another way to evaluate differences in theoretical orientation and TCIT, percentages of those who reported never having cried in therapy were compared. Although $49.9 \%$ of CBT clinicians reported never having cried in therapy, only $21.1 \%$ of the aggregated-dynamic group of clinicians reported never having cried (specifically, $21.1 \%$ of psychodynamic clinicians, $22.1 \%$ of clinicians with an eclectic orientation with a dynamic emphasis, and $0 \%$ of psychoanalytic clinicians report never having cried in therapy).

\section{Does Clinical Experience Impact TCIT?}

Significant differences were found between the crying tendency of graduate students and licensed psychologists, with graduate students reporting significantly lower crying tendency ( $n=324$, $M=1.82, S D=0.770)$ when compared with licensed psychologists $(n=226, M=2.20, S D=0.712 ; F=19.024, d f=2, p<$ $.001, d=0.51)$. When level of experience was operationalized as actual number of years in practice, TCIT Tendency significantly and positively correlated with increased clinical experience $(r=$ $.208, p<.001, N=568)$, as did TCIT Proneness $(r=.118, p=$ $.023, N=369$ ), such that the tendency and proneness to cry in therapy increased with the number of years in practice. Indeed, this trend is captured in a quotation from one of the participants: "I think the following reduce emotional tension felt about crying: the years of experience, age or life experience, and therapist training that includes therapy."

To gain another perspective of how experience may influence TCIT, age was analyzed in relation to TCIT Tendency and Proneness. Age significantly and positively correlated with TCIT Tendency $(r=.297, p<.001, N=554)$ and TCIT Proneness $(r=$ $.195, p<.001, N=365)$, such that the older the respondent, the 
Table 4

Theoretical Orientation and TCIT Frequency Ratio

\begin{tabular}{lcr}
\hline \multicolumn{1}{c}{ Theoretical orientation } & TCIT frequency ratio & $n$ \\
\hline Psychoanalytic & 0.033 & 9 \\
Eclectic with dynamic emphasis & 0.019 & 155 \\
Psychodynamic & 0.016 & 76 \\
Cognitive-behavioral & 0.013 & 232 \\
\hline
\end{tabular}

greater their tendency and proneness to cry in therapy. Interestingly, this trend was in the opposite direction of the (ACI) tendency and proneness to cry in daily life, where crying tendency $(r=-.116, p=.005, N=591)$ and proneness $(r=-.156, p<$ $.001, N=595)$ significantly decreased with age. When the ACI Proneness scale was broken down, the ACI Negative Affect Proneness scale significantly negatively correlated with age $(r=-.213$, $p=.001, N=595$ ), whereas the ACI Positive Affect Proneness scale significantly positively correlated with age $(r=.101, p=$ $.014, N=595)$. Overall, although tendency and proneness to cry in daily life (ACI) decreased with age, crying tendency and proneness in therapy (TCIT) increased with age.

\section{Therapeutic Effects of TCIT}

All respondents, whether or not they reported having cried in therapy, were provided with a list of possible therapeutic effects of TCIT and asked to check all those that they believed were likely effects of TCIT. "Likely" was qualified by the statement "you believe that in many instances this will occur." Those positive outcomes that more than half of all respondents believed to be "likely" consequences of TCIT are listed in Table $5(N=570)$. Negative outcomes that more than half of all respondents believed to be "likely" consequences of TCIT are listed in Table $6(N=$ 570).

Those respondents who reported that they had previously cried in therapy were asked to answer the question "Do you think your crying changed your relationship with your client?" Although $53.5 \%(n=191)$ of respondents indicated that nothing changed in their relationship with their client owing to their tears, $45.7 \%(n=$ 163 ) of respondents reported that their relationship improved owing to TCIT. Less than $1 \%(n=3)$ of respondents reported that their relationship became worse owing to TCIT. Of note, although the majority $(66.87 \%)$ of dynamic therapists reported relationship improvement due to TCIT, only $29.4 \%$ of CBT therapists did so,

Table 5

Likely Positive Effects of TCIT

\begin{tabular}{lcc}
\hline Expected positive consequence of TCIT & $\%$ & $n$ \\
\hline $\begin{array}{l}\text { The client feeling that the therapist genuinely } \\
\quad \text { cares about him/her }\end{array}$ & 82.28 & 469 \\
$\begin{array}{l}\text { Increased authenticity in the therapeutic } \\
\quad \text { relationship }\end{array}$ & 71.93 & 410 \\
Gives the client permission to feel & 60.70 & 346 \\
Models appropriate emotional expression & 58.95 & 336 \\
Increased openness in the therapeutic relationship & 58.77 & 335 \\
The client feeling that the therapist truly & 58.60 & 334 \\
$\quad$ understands him/her & & \\
Better rapport & 56.49 & 322 \\
\hline
\end{tabular}

Table 6

Likely Negative Effects of TCIT

\begin{tabular}{lcc}
\hline Expected negative consequence of TCIT & $\%$ & $\mathrm{n}$ \\
\hline $\begin{array}{l}\text { The client being concerned that the therapist will } \\
\text { not be able to handle the client's emotions }\end{array}$ & 69.12 & 394 \\
$\begin{array}{l}\text { The client feeling burdened by the therapist's } \\
\quad \text { emotion }\end{array}$ & 64.21 & 366 \\
$\begin{array}{l}\text { A role reversal in which the client will feel he/she } \\
\text { must take care of } \\
\text { the therapist }\end{array}$ & 56.32 & 321 \\
\hline
\end{tabular}

with the majority of CBT therapists $(69.6 \%)$ instead reporting that nothing changed.

\section{Discussion}

\section{Do Therapists Cry in Therapy?}

Seventy-two percent of the therapists in the present study reported that they had cried in therapy in their role as a therapist. This statistic can be compared with the sole statistic available regarding TCIT from the study by Pope et al. (1987) in which $56.5 \%$ of psychologists reported that they had cried in the presence of a client. Thus, the present study found a higher rate of therapists to have cried in the presence of a client than Pope et al.'s (1987) sample. There are several possible explanations for this. For one, crying was operationalized differently in the present study, such that it explicitly involved "tears in one's eyes due to emotional reasons," whereas Pope et al. (1987) did not define crying. In addition, ideas about neutrality in therapy (Aron, 1996; Bernstein, 1999) and emotional expression, in general, may have changed in the past 30 years, possibly influencing self-reported TCIT rates. Notably, a study of medical students and interns found that $68 \%$ of medical students and $74 \%$ of medical interns had cried with patients (Sung et al., 2009), reflecting a similar rate of crying among physicians as among the therapists in the present study.

\section{Do "Criers" Cry in Therapy?}

Results from the present study suggest that if a clinician is a "crier" in general, such that they have a higher tendency, proneness, and frequency of crying in daily life, the clinician is more likely to cry in therapy. This concept was echoed in an op-ed article on tears of medical doctors written since the present study completed data collection (May, 2011). Palliative care physician Christian Sinclair asserts that whether a physician cries with a patient is a matter of comfort with public displays of emotion.

\section{Does Gender Matter?}

The finding that sex of the clinician does not appear to relate to whether the clinician cries in therapy, nor with the frequency of tears in therapy, is surprising given that men cry significantly less than women in daily life, as demonstrated consistently in the literature (Bylsma, Vingerhoets, \& Rottenberg, 2008; Hastrup, Baker, Kraemer, \& Bornstein, 1986; Vingerhoets, Cornelius, Van Heck, \& Becht, 2000; Wagner et al., 1997), and as replicated in the present sample (in which women cried more than twice as much as 
men in daily life: the woman-to-man ratio of crying frequency in daily life was 2.2:1 (women: $n=464, M=3.08, S D=0.731$; men: $n=142, M=2.38, S D=0.741)$, and Student's $t$ test found this difference to be significant ( $p<.001, d=0.95$ ). An explanation for this lack of sex difference in TCIT may be found in women's higher reported rates of feeling the urge to cry and inhibiting their tears in therapy. In other words, women may hold back tears more often in therapy than in daily life, as compared with men, and thus, their rates of TCIT are more comparable with men, who are less likely to inhibit their tears, by their report. It may also be that men, who are less likely to report inhibiting their tears in therapy than women, are doing exactly that: inhibiting their tears in therapy less, perhaps, than in daily life, relative to women. Thus, they are perhaps allowing themselves to tear up and cry more frequently in therapy than they would in daily life outside the therapy setting.

It is also possible that gender role expectations for men regarding emotional expression apply less in the therapy situation. Many men experience difficulty in expressing emotion, that is, normative male alexithymia (Levant, 2001), a phrase that refers to the pervasive socialization process by which men learn-from parents, peers, and other men-to suppress the expression of feelings. Thus, male therapists are, in some ways, going "against the norm" of male alexythmia simply in their role as a therapist, a role that requires attention to, and ability in, expressing emotion states. When in therapy, men may be less likely to adhere to strict gender role expectations regarding emotional expression, perhaps even as a means of modeling appropriate affect. Indeed, men were more likely than women to say that "modeling appropriate emotional expression" is a likely consequence of TCIT. In addition, men in this study, as well as in previous studies (Trezza, Hastrup, \& Kim, 1988), report higher average crying frequency in daily life than the general population (Hastrup et al., 1986), suggesting that male therapists may go against strict gender role expectations in terms of emotional displays not only in therapy but also in daily life.

Another possible explanation for what it is that makes the gender difference in crying in daily life disappear in therapy may be related to the unique affective tone of therapy sessions in which TCIT occurs. Respondents in the present study were presented with a list of emotions and asked to check which they felt during their most recent crying episode (they could check more than one). The most common emotion reported was sadness $(75 \%)$, the second most common emotional state was feeling emotionally "touched" (63\%), and the third was warmth (33\%). Furthermore, the fifth most common emotion was gratitude (15\%), and the seventh was joy $(12 \%)$. Thus, of the top three most common emotional experiences, two were generally "positive" affect states, and one was "negative" (i.e., sadness). Of the top seven, four were "positive," and three were "negative." This is in contrast to the top three emotions felt by the client, as speculated by the therapist: sadness (59\%), grief/loss (38\%), and powerlessness (34\%), all "negative" emotions. This suggests that TCIT may occur in situations in which the therapist experiences a more positive affective valence than one might intuitively assume. Indeed, in $73.7 \%$ of most recent crying episodes, respondents checked at least one positive emotion. In $54.6 \%$, at least one positive and one negative emotion were listed, indicating an often "mixed" emotional valence for the therapist.
And why might the positive affective tone help to explain the lack of gender difference in TCIT? Previous research has found that when men cry in daily life, they tend to cry more owing to positive than to negative affect (i.e., men cry more at weddings than at funerals; Becht \& Vingerhoets, 1997; Bindra, 1972; Bylsma et al., 2008). In the present study, although women showed higher rates of crying in daily life in terms of ACI crying tendency, frequency, and proneness, the difference between men and women was smallest on the ACI Positive Affect Proneness scale, a scale measuring the proneness to cry owing to positive affect, suggesting that this trend holds true in the present sample. If we thus understand that men cry more in situations with a positive affective valence than a negative affective valence, it is possible that the relatively higher rates of TCIT among men are explained by a more positive (or mixed) affective tone in therapy sessions in which TCIT occurs.

In short, the well-document trend-in which women cry more than men in daily life-held true in this study (as measured by the ACI), but did not hold true in therapy (i.e., TCIT). This suggests that tears that occur in the therapy situation are different in nature than tears shed in daily life.

\section{Does Personality Matter?}

Overall, although it is possible that clinicians who are more open, agreeable, and extraverted may be slightly more likely to cry in therapy, these findings were equivocal and should be followed up in research with a more nuanced personality inventory. It is notable that clinicians who were more neurotic (defined by the tendency to experience negative emotionality; John \& Srivastava, 1999) tended to cry more in daily life, a finding demonstrated in previous studies (De Fruyt, 1997; Vingerhoets et al., 2000; Vingerhoets \& Van Heck, 1993) and replicated in the present study. However, the relationship between neuroticism and TCIT is less clear. This suggests that, perhaps, the tendency to experience negative emotions (the definition of neurotic) that appears to correlate with crying in daily life may not be associated with crying in therapy. Thus, the affective valence that leads "neurotics" to cry in daily life may differ from the affective valence leading to TCIT, implying, as described earlier in the text, that a more positive affective tone for the therapist may accompany TCIT. Along this same line of thinking, the trait of openness, which, of all Big Five traits, correlated most strongly with TCIT Tendency, only correlated with the ACI Positive Affect Proneness scale $(r=.109, p=.007, N=610)$ and not at all with the ACI Negative Affect Proneness scale (i.e., "open” respondents were slightly more likely to cry owing to positive affect in daily life as well as more likely to cry in therapy). Similarly, the trait of extraversion, which demonstrated a small positive correlation with TCIT Tendency, very slightly negatively correlated with overall ACI Frequency $(r=-.09, p=.036, N=606)$, but positively correlated with the ACI Positive Affect Proneness scale $(r=.104, p=.01, N=610)$. Although these correlations are small, it does appear that extraversion positively correlates with crying in daily life owing to positive affect as well as crying in therapy, but negatively correlates with crying frequency in daily life overall. 


\section{Does Empathy Make Us Cry?}

Part of the reason the relationship between empathy and TCIT remains equivocal may be due to the measure of empathy used: the TEQ, which is designed as a unidimensional measure of empathy. It is possible that particular traits within the overarching concept of empathy correlate more specifically with TCIT. Indeed, empathy may often be present during a therapy session, and thus, specific aspects of empathy may be predictive of TCIT specifically. Future research may benefit from using another measure of empathy designed to distinguish between various aspects of empathy (i.e., cognitive, affective), for example, the Basic Empathy Scale (Jolliffe \& Farrington, 2006).

\section{Theoretical Orientation and TCIT}

It is curious to note that although there was a clear significant difference in TCIT rates between CBT and dynamically oriented clinicians, there were no significant differences found between CBT and dynamically oriented therapist on the ACI scales. In other words, although CBT clinicians report crying significantly less than dynamically oriented clinicians in therapy, the two groups cry at similar rates in daily life. This suggests (again) that something about the therapy situation specifically leads to differences in rates of crying among these groups. In their outline of features that distinguish dynamic therapies from other therapies, Blagys and Hilsenroth (2000) may provide some explanation for this. The first distinguishing feature of psychodynamic therapy that they list is a "focus on affect and expression of patient's emotion" (p. 169). In other words, the therapist hones in on the emotive material, thus perhaps evoking more crying-inducing session content than would other techniques. Perhaps it is this focus on emotion and emotional expression in dynamic therapies, and the possible result of more crying-inducing material, that leads dynamic therapists to feel and express more affect themselves while conducting therapy and, as one form of emotional expression, to cry more in therapy.

Blagys and Hilsenroth (2000) also note that dynamic therapies are distinguished by their "emphasis on the therapeutic relationship" (p. 181). Such a focus on the process occurring between therapist and client may foster a sense of connection, emotional intensity, and/or possibly the tendency for the therapist feel positively touched by the patient, all of which may lead to increased TCIT among dynamic clinicians.

\section{Does Clinical Experience Impact TCIT?}

Data from the present study suggest that the more time spent conducting therapy (i.e., the more years in practice), the more one cries in therapy. Data also suggest that older respondents have higher TCIT rates. To understand the relationship between clinical experience and age more clearly, it is helpful to note that older respondents in the present study had a lower tendency and proneness to cry in daily life, as measured by the ACI, a trend found in other studies as well (Borquist, 1906; Williams \& Morris, 1996). Because the tendency and proneness to cry in therapy (TCIT) increased with age, it is notable that the direction of the correlation reversed. This reversal suggests that something about crying in therapy specifically is related to age, as greater tendency to cry in general is not reported by older respondents. It may be that clinical experience acts as a mediating variable in the relationship between TCIT and age. Indeed, the trend found in the present study-in which TCIT increased with clinical experience-is similar to trends found in other studies. For instance, in studying tears in the medical profession, Wagner et al. (1997) found that doctors were proportionally more likely to cry than medical students $(57 \%$ of doctors had cried, whereas $31 \%$ of medical students had cried). In a dissertation on TCIT with psychodynamic clinicians (Waldman, 1995), several participants expressed a sense that they had become more comfortable with their own tears over the years of their practice. The one participant in that study who was conflicted about his tears was also the least-experienced practitioner.

It is also possible that, with experience, therapists become more comfortable setting flexible boundaries, or in using their clinical judgment in being less rigid, and thus, more experienced therapists have fewer restrictions on their own affective displays. A process such as this seems to be the case, for instance, with therapist self-disclosure. Constance (2008) examined differences between psychologists' and trainees' beliefs about self-disclosure and found that psychologists believed self-disclosure to be more ethical than trainees on the majority of her survey items. Similarly, in a dissertation on the topic, Gallucci (2002) found that highly experienced therapists disclosed more frequently than novice therapists. Although the relationship between TCIT and self-disclosure is not entirely clear, it is possibly that the findings from these self-disclosure studies are relevant to TCIT as well. Thus, more experienced therapists may cry more frequently in therapy, just as they self-disclose more frequently.

The affective valence of therapy sessions in which TCIT occurs may provide additional insight into the relationship between age, clinical experience and TCIT. As described in the results section, although the total ACI Proneness scale negatively correlated with age (i.e., the older the respondent, the lower their crying proneness), only the Negative Affect Proneness scale significantly negatively correlated with age (i.e., as age increased, the number of crying episodes due to negative affect decreased). The correlation between the Positive Affect Proneness scale of the ACI also significantly correlated with age, but the direction of the correlation reversed and was positive (i.e., as age increased, the number of crying episodes due to positive affect increased). Thus, like the TCIT Tendency and TCIT Proneness scales, the ACI Positive Affect Proneness scale significantly positively correlated with age. In other words, older individuals cry more in daily life owing to positive affect situations but less in daily life owing to negative affect situations. We may again speculate that the affective tone of scenarios in which clinicians-particularly older clinicians - cry in therapy may be more "positive" or "mixed" than the general scenarios in which individuals cry in their own day-to-day lives, thus contributing to increased TCIT rates among older clinicians.

\section{Therapeutic Effects of TCIT}

Based on the results of the present study, it appears that when TCIT had a positive outcome (from the therapist's perspective), it was primarily through its impact on the therapeutic relationship, and the perception of caring and authenticity between client and therapist. It is also noteworthy that, although just over half $(53.5 \%)$ of respondents felt TCIT had no real impact, for better or worse, on 
the therapeutic relationship, nearly half $(45.7 \%)$ said that the relationship changed for the better owing to TCIT — a fairly high percentage reporting a positive outcome. Less than $1 \%$ reported a negative change. In writing of bedside crying among physicians, author Christian Sinclair describes a similar effect: "No one seemed hurt or bothered by the (crying of doctors), and in fact some of the connections were much stronger because of the shared experience" (2011).

The potential for TCIT to have a negative impact was believed by respondents to be largely in the possible role reversal it can cause. Again, Sinclair (2011) offers a similar caution to doctors: "What a professional should not do is allow the crying to change the focus from the family or patient to the physician."

But when might TCIT have a positive impact, and when might its impact go awry? Again, perhaps literature on therapist selfdisclosure could apply to TCIT. In their study of the impact of self-disclosure on the therapeutic relationship, Myers and Hayes (2006) found that therapy sessions were rated (by independent observers) to be "deeper" and the therapists to be more skilled when the therapist made disclosures, but only when there was a positive therapeutic alliance in place. The reverse was true when the alliance was negative: disclosures were seen as "shallow" and a sign of less expertise by the therapist. While speculative, perhaps a similar process occurs with TCIT, such that TCIT has a positive impact when it can deepen an already strong and positive rapport, but can pose further threat to an alliance that is weak or negative.

\section{Limitations}

This study was based on a largely Caucasian (80\%) sample and a relatively young group of clinicians, both of which impact generalizability. The study was based on self-report, with many of the questions being face valid. Another study limitation is possible selection bias. Although the researcher made efforts to disguise the emphasis on TCIT by describing the research as a study on therapist emotions in therapy, it is possible that those individuals who cry were more likely to participate in the study, thus elevating the rates of crying, in general, and TCIT. Additionally, several outcome-oriented questions (i.e., "Do you think your crying changed your relationship with your client") were asked. However, no data were collected from the client or from an outside observer. Finally, future research may benefit from the use of a more sensitive personality measure as well as a multidimensional measure of empathy, to potentially capture more nuanced data regarding the relationship between TCIT, personality, and empathy.

\section{Conclusion}

In summary, TCIT happens. Indeed, the majority of clinicians are likely to experience tearing up or crying in therapy at some point in their career. Although the purpose of this study was not to give therapists permission to cry, or conversely, to impose some kind of a moratorium against TCIT, it may be de-shaming for those who have cried or will cry in therapy to know that they are not alone (i.e., they are in the $72 \%$ majority). It may also be helpful for clinicians to note that certain traits - such as sex, personality, and even empathy - do not appear to influence whether a clinician cries in therapy. Instead, there are important contextual factors that do appear to influence TCIT rates, factors related to unique aspects of the therapy itself and the therapist's identity in the therapeutic context (i.e., clinical experience, theoretical orientation, affective tone of the session). And although the purpose of the present study was not to characterize TCIT as appropriate or inappropriate, ethical or unethical, therapeutically constructive or detrimental, it is worth noting that the majority of those who reported having cried in therapy did not report negative consequences of their tears. In fact, almost half of therapists reported improvement in the therapeutic relationship that they attributed to TCIT. Also noteworthy is the finding that greater clinical experience was related to greater acknowledgment of crying by therapists, indicating that tears are more common among "experts." Perhaps with greater experience, therapists feel more comfortable in allowing themselves to experience such emotions (and/or to report as much in a survey), or perhaps, more experienced clinicians recognize the value of TCIT and/or believe it not to be of harm.

It does appear that individuals who are "criers" in daily life, such that they report higher scores on the ACI, report higher TCIT scores, suggesting that this interindividual trait-that is, being a "crier"-may influence the likelihood of crying in therapy. However, other demographic-type factors in general do not appear to predict TCIT. For example, although men cry less than women in daily life- both in previous research and in the present studythere are no significant gender differences in TCIT. Furthermore, personality traits do not appear to be highly influential in whether-or how often - a therapist cries. Indeed, neuroticism, which has been found to positively correlate with higher rates of crying in daily life both in previous research and in the present study, does not appear to significantly correlate with TCIT. Empathy has been documented to positively correlate with crying in daily life, both in previous research (Choti, Marston, Holston, \& Hart, 1987; Vingerhoets et al., 2000) and in the present study, but the relationship between TCIT and empathy remains equivocal. Theoretical orientation did not significantly correlate with crying in daily life, but there were significant differences in TCIT trends between theoretical orientations, with psychodynamic clinicians reporting higher rates of TCIT than CBT clinicians. And although increasing age predicts less crying in daily life in the present sample, older clinicians cry more in therapy, just as more experienced clinicians cry more than trainees (which may help to explain the relationship between age and TCIT). Thus, the variables we may use to predict tears in daily life appear to differ from those with which we may predict tears in therapy. One possible reason for this that has been put forth earlier in the text is that the affective tone of therapy sessions - or moments - in which TCIT occurs is more positive or emotionally "mixed" than situations in which individuals tend to cry in daily life. This is meaningful, as it challenges a potential view of TCIT as occurring due to the therapist being overwhelmed by intense negative emotions that arise in therapy, and instead signals a moment of potentially positive emotional connection, even if amid painful negative affect.

\section{Future Research}

Very little research has been conducted on the topic of TCIT. This study represents the first large-scale mixed-method research project to thoroughly investigate TCIT in U.S. psychologists and trainees. Future research would do well to investigate the effects of TCIT on the therapeutic relationship and therapy outcomes, as 
such work may have implications for the ways in which therapists manage their own affect in session. Future research should also focus on the clients' perspectives of TCIT. Although this study unintentionally gathered some information on clients' perspectives (i.e., participants volunteered descriptions of their experiences of being a client in therapy when their therapist cried), as the following quotation demonstrates, no systematic assessment was conducted.

"Before I became a psychologist and while I was in my own private individual therapy, my therapist teared up once while I shared my most painful childhood memory. I was deeply touched by this, I felt closer to her, and I appreciated her empathy. This impacted my own later respect for the rare but meaningful moments when I, too, would get teary with my patients."

\section{References}

Alden, P. A. (2001). Gently wiping her tears away. In S. Kahn \& E. Fromm (Eds.), Changes in the therapist (pp. 117-131). Mahwah, NJ: Erlbaum Publishers.

Aron, L. (1996). A meeting of minds: Mutuality in psychoanalysis. Hillsdale, NJ: Analytic Press.

Barth, A., Egger, A., Hladschik-Kermer, B., \& Kropiunigg, U. (2004). Shedding tears in hospitals: A survey of medical staff and students. Psychotherapie, Psychosomatik, Medizinische Psychologies, 54, 194197. doi:10.1055/s-2003-814865

Becht, M. C., Poortinga, Y. H., \& Vingerhoets, A. J. (2001). Crying across countries. In A. J. Vingerhoets \& R. R. Cornelius (Eds.), Adult crying: A biopsychosocial approach (pp. 277-298). Hove, UK: BrunnerRoutledge.

Becht, M., \& Vingerhoets, A. J. (1997, March). Why we cry and how it affects mood. Paper presented at the annual meeting of the American Psychosomatic Society, Santa Fe, NM.

Bernstein, J. W. (1999). The politics of self-disclosure. Psychoanalytic Review, 86, 595-605.

Bindra, D. (1972). Weeping, a problem of many facets. Bulletin of the British Psychological Society, 25, 281-284.

Blagys, M. D., \& Hilsenroth, M. J. (2000). Distinctive features of shortterm psychodynamic- interpersonal psychotherapy: A review of the comparative psychotherapy process literature. Clinical Psychology: Science and Practice, 7, 167-188. doi:10.1093/dinsy/7.2.167

Blankenship, V. A. (1984). A comparative study of student nurses, nursing faculty, and staff nurses in their perceptions of weeping, their weeping behaviors and their interventions with the weeping patient (Doctoral dissertation). Retrieved from Dissertations and Theses database. (UMI No. 8508252 )

Blume-Marcovici, A. C. (2012). Tracking our tears: An empirical investigation of therapist crying in therapy (Doctoral dissertation). Retrieved from Dissertation and Theses database. (UMI No. 3503469)

Borquist, A. (1906). Crying. The American Journal of Psychology, 17, 149-205.

Bylsma, L. M., Vingerhoets, A. J., \& Rottenberg, J. (2008). When is crying cathartic? An international study. Journal of Social and Clinical Psychology, 27, 1165-1187. doi:10.1521/jscp.2008.27/10/1165

Choti, S. E., Marston, A. R., Holston, S. G., \& Hart, J. T. (1987). Gender and personality variables in film-induced sadness and crying. Journal of Social and Clinical Psychology, 5, 535-544.

Cohen, J. (1988). Statistical power analysis for the behavioral sciences (2nd ed.). Hillsdale, NJ: Erlbaum.

Constance, J. E. (2008). Self-disclosure behaviors of psychologists: A comparison of psychologists and psychological trainees across specialties (Doctoral dissertation). Retrieved from Dissertations and Theses database. (UMI No. 3351877)
Counselman, E. F. (1997). Self-disclosure, tears, and the dying client Psychotherapy, 34, 233-237. doi:10.1037/h0087715

Cusi, A. M., MacQueen, G. M., Spreng, R., \& McKinnon, M. C. (2011). Altered empathic responding in major depressive disorder: Relation to symptom severity, illness burden and psychosocial outcome. Psychiatry Research, 188, 231-236. doi:10.1016/psychres.2011.04.013

De Fruyt, F. (1997). Gender and individual differences in adult crying. Personality and Individual Differences, 22, 937-940. doi:10.1016/ S0191-8869(96)00264-4

Gallucci, A. (2002). Therapists' use of self-disclosure: A quantitative study (Doctoral dissertation). Retrieved from Dissertation and Theses database. (UMI No. 3052691)

Gosling, S. D., Rentfrow, P. J., \& Swann, W. B. (2003). A very brief measure of the Big- Five personality domains. Journal of Research in Personality, 37, 504-528. doi:10.1016/S0092-6566(03)00046-1

Guntrip, H. (1986). My experience of analysis with Fairbairn and Winnicott. In P. Buckley (Ed.), Essential papers on object relations (pp. 447-468) (Original work published in 1975). New York: New York University Press.

Hastrup, J. L., Baker, J. G., Kraemer, D. L., \& Bornstein, R. F. (1986). Crying and depression among older adults. The Gerontologist, 26, 9196. doi:10.1093/geront/26.1.91

Hemphill, J. F. (2003). Interpreting the magnitudes of correlation coefficients. American Psychologist, 58, 78-80. doi:10.1037/0003-066X.58 1.78

John, O. P., \& Srivastava, S. (1999). The big five trait taxonomy: History, measurement, and theoretical perspectives. In L. A. Pervin \& O. P. John (Eds.), Handbook of personality: Theory and research (pp. 102-138). New York: Guilford Press.

Jolliffe, D., \& Farrington, D. P. (2006). Development and validation of the basic empathy scale. Journal of Adolescense, 29, 589-611. doi:10.1016/ j.adolescence.2005.08.010

Kahn, S., \& Fromm, E. (Eds.). (2001). Changes in the therapist. Mahwah, NJ: Erlbaum.

Krauser, P. S. (1989). Tears. "A piece of my mind” entry essay. Journal of the American Medical Association, 261, 3612

Kukulu, K. (2006). Patient crying in hospitals: A survey on undergraduate nursing and medical students. Medical Teacher, 28, 487.

Labott, S. M. (2001). Crying in psychotherapy. In A. J. Vingerhoets \& R. R. Cornelius (Eds.) Adult crying: A biopsychosocial approach (pp. 213-226). Hove, UK: Brunner-Routledge.

Lerner, B. H. (2008, April 22). At bedside, stay stoic or display emotions? New York Times. Retrieved from http://www.nytimes.com/2008/04/22/ health/views/22essa.html

Levant, R. F. (2001). Desperately seeking language: Understanding, assessing, and treating normative male alexithymia. In G. R. Brooks \& G. E. Good (Eds.), The new handbook of psychotherapy and counseling with men: A comprehensive guide to settings, problems, and treatment approaches (Vols. 1 \& 2, pp. 424-443). San Francisco, CA: JosseyBass.

Majhail, N. S., \& Warlick, E. D. (2011). To cry or not to cry: Physicians and emotions at the bedside. Minnesota Medicine, 94. Retrieved from http://www.ncbi.nlm.nih.gov/pubmed/21366107.

Mayotte-Blum, J., Slavin-Mulford, J., Lehmann, M., Pesale, F., BeckerMatero, N., \& Hilsenroth, M. (2012). Therapeutic immediacy across long-term psychodynamic psychotherapy: An evidence-based case study. Journal of Counseling Psychology, 59, 27-40.

McWilliams, N. (1994). Psychoanalytic diagnosis. New York: Guilford Press.

Myers, D., \& Hayes, J. A. (2006). Effects of therapist general selfdisclosure and countertransference disclosure on ratings of the therapist and session. Psychotherapy: Theory, Research, Practice, Training, 43, 173-185. doi:10.1037/0033-3204.43.2.173 
Nelson, J. K. (2008). Crying in psychotherapy: Its meaning, assessment, and management based on attachment theory. In A. J. J. M. Vingerhoets, I. Nyklicek, \& J. Denollet (Eds.), Emotion regulation and health. Conceptual and clinical issues (pp. 202-214). New York, NY: Springer.

Owens, C. (2005). Moved to tears: Technical considerations and dilemmas encountered in working with a 13-year-old boy with acquired quadriplegia. Journal of Child Psychotherapy, 31, 284-302. doi:10.1080/ 00754170500370753

Pope, K. S., Tabachnick, B. G., \& Keith-Spiegel, P. (1987). Ethics of practice: The beliefs and behaviors of psychologists as therapists. American Psychologist, 42, 993-1006. doi:10.1037/0003-066X.42.11.993

Rhue, J. W. (2001). Death, bereavement, and the therapist. In S. Kahn \& E. Fromm (Eds.), Changes in the therapist (pp. 117-131). Mahwah, NJ: Erlbaum Publishers.

Scheirs, J. G. M., \& Sijtsma, K. (2001). The study of crying: Some methodological considerations and a comparison of methods for analyzing questionnaires. In A. J. J. M., Vingerhoets \& R. R. Cornelius (Eds.), Adult crying: A biopsychosocial approach (pp. 277-298). Hove, UK: Brunner-Routledge.

Sinclair, C. T. (2011). To cry or not to cry at the bedside. Medscape News Today. Retrieved from http://www.medscape.com/viewarticle/ 741508?src=nl_topic

Spreng, R. N., McKinnon, M. C., Mar, R. A., \& Levine, B. (2009). The Toronto Empathy Questionnaire: Scale development and initial validation of a factor-analytic solution to multiple empathy measures. Journal of Personality Assessment, 91, 62-71. doi:10.1080/00223890802484381

Summers, R. F., \& Barber, J. P. (2010). Psychodynamic therapy: A guide to evidence- based practice. New York: The Guilford Press.

Sung, A. D., Collins, M. E., Smith, A. K. Sanders, A. M., Quinn, M. A., Block, S. D., \& Arnold, R. M. (2009). Crying: Experiences and attitudes of third-year medical students and interns. Teaching and Learning in Medicine, 21, 180-187. doi:10.1080/10401330903014111

Trezza, G. R., Hastrup, J. L., \& Kim, S. E. (1988). Clinicians' attitudes and beliefs about crying behavior. Paper presented at Fifty-ninth Annual Meeting of the Eastern Psychological Association, Buffalo, NY.

Van Heukelem, J. F. (1979). Weep with those who weep: Understanding helping the crying person. Journal of Psychology and Theology, 7, 83-91.

Vingerhoets, A. J., \& Cornelius, R. R. (2001). Adult crying: A biopsychosocial approach. Hove, UK: Brunner-Routledge.

Vingerhoets, A. J., Cornelius, R. R., Van Heck, G. L., \& Becht, M. C. (2000). Adult crying: A model and review of the literature. Review of General Psychology, 4, 354-377. doi:10.1037/1089-2680.4.4.354

Vingerhoets, A. J., \& Van Heck, G. L. (1993). The psychobiological aspects of stress and emotions: Some alternative views. In U. Hentschel \& E. H. M. Eurelings-Bontekoe (Eds.), Experimental research in psychosomatics (pp. 19-36). Leiden, The Netherlands: DSWO Press.

Wagner, R. E., Hexel, M., Bauer, W. W., \& Kropiunigg, U. (1997). Crying in hospitals: A survey of doctors', nurses' and medical students' experience and attitudes. Medical Journal of Australia, 166, 13-16.

Waldman, J. L. (1995). Breakthrough or breakdown: When the psychotherapist cried during the therapy session (Doctoral dissertation). Retrieved from Dissertation and Theses database. (UMI No. 9536358)

Williams, D. G., \& Morris, G. H. (1996). Crying, weeping or tearfulness in British and Israeli adults. British Journal of Psychology, 87, 479-505. doi:10.1111/j.2044-8295.1995.tb02603.x

Received November 20, 2012 Accepted November 21, 2012

\section{E-Mail Notification of Your Latest Issue Online!}

Would you like to know when the next issue of your favorite APA journal will be available online? This service is now available to you. Sign up at http://notify.apa.org/ and you will be notified by e-mail when issues of interest to you become available! 